\title{
SIMULTANEOUS EXTENSIONS FROM DISCRETE SUBSPACES
}

\author{
H. BANILOWER
}

\begin{abstract}
If $N$ is a denumerable, discrete, normally embedded subspace of the completely regular space $S$, then any bounded linear operator from $C(N)$ into $C(S)$ that extends functions in $C_{0}(N)$ necessarily extends all bounded functions on some infinite subset of $N$ (that are zero elsewhere on $N$ ). For compact $S$, such operators exist whenever $C(S)$ contains a subspace isometric to $(m)$. It is also shown, assuming the continuum hypothesis, that if $S$ is a locally compact $F$-space and $C_{0}(S)$ is complemented in $C(S)$, then $S$ is countably compact.
\end{abstract}

Introduction. The main results of $\S 1$ are Theorems 1.1 and 1.5 , which are precise formulations of the first two statements of the abstract. This section also contains a previously unpublished result, Proposition 1.2, of H. P. Rosenthal which appears here with his permission. It states that any operator on $(m)$ having norm one which is an isometry on $\left(c_{0}\right)$ is necessarily an isometry on $(m)$.

In $\S 2$, we assume the continuum hypothesis, denoted $[\mathrm{CH}]$, and that $S$ is an $F$-space. Our main result here is Theorem 2.5 , which is the final sentence of the abstract. This improves previous results of W. W. Comfort and the author which, however, do not assume [CH].

We would like to thank the referee for bringing Rosenthal's result to our attention and for pointing out its utility in simplifying the proof of Theorem 1.5 and in strengthening Theorem 2.3 compared to their original versions.

Background. We give [DS] and [GJ] as general references. Familiarity with [B] and the references therein is helpful but not essential.

$S$ always denotes a completely regular Hausdorff space and $N$ a countably infinite discrete (in the relative topology) subspace of $S . C(S)$ is the Banach space of all bounded continuous real or complex valued functions on $S$ with supremum norm.

Let $A$ be a subspace of $S$.

Presented to the Society, February 5, 1971; received by the editors April 5, 1971 and, in revised form, April 13, 1972.

AMS 1970 subject classifications. Primary 46E15, 54G05; Secondary 54C45.

Key words and phrases. Simultaneous extension, discrete subspace, F-space, subspace isometric to $(m)$.

(c) American Mathematical Society 1973 
$A$ is normally embedded in $S$ is every element of $C(A)$ has a continuous extension to $S$. If so, we may identify $\beta A$ with $\mathrm{cl}_{\beta S} A$ where $\beta S$ is the StoneČech compactification of $S$.

If $X$ and $Y$ are Banach spaces, the notation $T: X \rightarrow Y$ means that $T$ is a bounded linear operator from $X$ into $Y . X$ is isomorphic (respectively isometric) to $Y$ if there exists a one-to-one bicontinuous (respectively norm-preserving) operator from $X$ onto $Y$.

If $X$ and $Y$ are subspaces of $C(A)$ and $C(S)$ respectively, a simultaneous extension is an operator $T: X \rightarrow Y$ satisfying $T f \mid A=f$ for all $f \in X$.

$C(S \| A)$ denotes the space of all functions in $C(S)$ which are zero on $A$.

We shall use without mention the natural isometry between $C(S)$ and $C(\beta S)$ and do not distinguish between an operator from $C(A)$ into $C(S)$ and its natural counterpart from $C(A)$ into $C(\beta S)$ (or, if $A$ is normally embedded in $S$, the one from $C(\beta A)$ into $C(\beta S))$.

For locally compact $S, C_{0}(S)$ is the subspace of functions in $C(S)$ that vanish at infinity. It is isometric to $C(\beta S \| \beta S-S)$.

$(m)$ denotes the Banach space of all bounded scalar valued sequences and $\left(c_{0}\right)$ its subspace of all sequences vanishing at infinity. They are isometric to $C(N)$ and $C_{0}(N)$, respectively, for any $N$.

1. Our first theorem is the analogue of a result of H. P. Rosenthal [R, Proposition 1.2] for general isomorphisms. Its proof was suggested by Remark 2 which follows his result.

THEOREM 1.1. If $N$ is normally embedded in $S, T: C(N) \rightarrow C(S)$, and $T \mid C_{0}(N)$ is a simultaneous extension, then there exists an infinite $M \subset N$ such that $T \mid C(M)$ is a simultaneous extension.

(We identify here $C(M)$ with the subspace $C(N \| N-M)$ of $C(N)$.)

Proof. Since $\beta N=\operatorname{cl}_{\beta S} N$, we may assume that $S$ is compact, $\beta N \subset S$, and $T: C(\beta N) \rightarrow C(S)$. To simplify notation denote the points of $N$ by $n$.

For each $n \in N$, define $\pi_{n}=T^{*} \delta_{n}$, where $\delta_{n}$ is the unit point mass at $n$ in the conjugate space of $C(S)$.

If $\chi_{k}$ denotes that element of $C(\beta N)$ that is the characteristic function of $\{k\}$, then by hypothesis we have $T \chi_{k}(n)=\chi_{k}(n)$ for all $k, n \in N$. Hence it follows from the definition of adjoint operator that $\pi_{n}(\{k\})=\chi_{k}(n)$.

Since $\beta N-N$ has an uncountable family of pairwise disjoint clopen sets and each $\pi_{n}$ is countably additive, there exists an infinite $M \subset N$ such that $\pi_{n}(\beta M-M)=0$ for all $n$.

Identifying $\beta M$ with $\mathrm{cl}_{\beta S} M$ and $C(\beta M)$ with $C(\beta N \| \beta N-\beta M)$, it follows that $\pi_{m}(f)=f(m)$ for all $m \in M$ and all $f \in C(\beta M)$.

Hence $T f(m)=\delta_{m}(T f)=f(m)$, and since $M$ is dense in $\beta M, T \mid C(\beta M)$ is a simultaneous extension. This completes the proof. 
In the following, which is Rosenthal's result, $B$ is an arbitrary Banach space and $N$ the set of positive integers. The proof is Rosenthal's.

Proposition 1.2. If $T:(m) \rightarrow B,\|T\|=1$, and $T \mid\left(c_{0}\right)$ is an isometry, then $T$ is an isometry. Moreover, if $B=(m)$ and $T\left|\left(c_{0}\right)=I\right|\left(c_{0}\right)$, then $T=I$.

Proof. By the Hahn-Banach theorem, we may choose linear functionals $f_{n}$ in $B^{*}$ of norm one such that $f_{n}(T \phi)=\phi(n)$ for all $n \in N$ and $\phi \in\left(c_{0}\right)$. Regarding $T^{*} f_{n}$ as a scalar valued Borel measure on $N$, we thus have that $\left(T^{*} f_{n}\right)\{n\}=1$, and since $T^{*}$ has norm one, $\left\|T^{*} f_{n}\right\|=1$; hence this measure must be supported on $\{n\}$, i.e., $T^{*} f_{n}=\delta_{n}$, whence $\left(T^{*} f_{n}\right)(\phi)=$ $\phi(n)$ for all $\phi \in(m)$. It follows immediately that $T$ is an isometry; the "moreover" part follows by observing that under these additional hypotheses, we may set $f_{n}=\delta_{n}$ for all $n$.

It will be convenient, for our applications, to state the simultaneous extension version of the above.

Corollary 1.3. If $T: C(N) \rightarrow C(S),\|T\|=1$, and $T \mid C_{0}(N)$ is a simultaneous extension, then $T$ is a simultaneous extension.

Proof. Let $R: C(S) \rightarrow C(N)$ be the restriction operator. Since $\|R T\|=1$ and $R T\left|C_{0}(N)=I\right| C_{0}(N)$, the conclusion follows immediately from Proposition 1.2.

For our next theorem we need the following result. It is proven in [G, Corollaries 1 and 2] and also follows directly from $[\mathbf{H}]$.

Proposition 1.4. If $S$ is compact and $U:(m) \rightarrow C(S)$ is an isometry, then $S$ contains a normally embedded $N$ such that

(i) $\left|U 1\left(t_{n}\right)\right|=1$, and

(ii) $U \alpha\left(t_{n}\right)=\alpha_{n} \cdot U 1\left(t_{n}\right)$, for all $\alpha \in(m), t_{n} \in N$.

THEOREM 1.5. For compact $S, C(S)$ contains a subspace isometric to $(m)$ if and only if there exists (a denumerable discrete) $N \subset S$ and a simultaneous extension $E: C(N) \rightarrow C(S)$, with $\|E\|=1$.

Proof. Since any simultaneous extension of norm one is an isometry, one implication is trivial.

To prove the other, let $U$ and $N$ be as in Proposition 1.4. Define $Q$ by the formula

$$
(Q g)_{n}=g\left(t_{n}\right) / U 1\left(t_{n}\right), \quad g \in C(N) .
$$

It is easily verified that $Q$ is an isometry from $C(N)$ onto $(m)$, and hence that $T=U Q$ is an isometry from $C(N)$ into $C(S)$.

For each $n$, let $g_{n} \in C(N)$ denote the characteristic function of $\left\{t_{n}\right\}$. 
Then for all $m, n$, we have $T g_{m}\left(t_{n}\right)=U\left(Q g_{m}\right)\left(t_{n}\right)=\left(Q g_{m}\right)_{n} \cdot T 1\left(t_{n}\right)=$ $g_{m}\left(t_{n}\right)$. Hence $T \mid C_{0}(N)$ is a simultaneous extension. Applying Corollary 1.3 completes the proof.

2. In this section we apply the results of $\S 1$ to the case where $S$ is an $F$-space. This class of spaces, which includes the extremally disconnected spaces but also contains connected spaces, is discussed in [GJ, Chapter 14]. We shall use the following characterization of $F$-spaces due to J. Lindenstrauss [L, p. 218].

Proposition 2.1 [CH]. $S$ is an F-space if and only if for every two Banach spaces $X \supset Y$ and every operator $T: Y \rightarrow C(S)$ with separable range, $T$ has an extension to $T^{\prime}: X \rightarrow C(S)$, with $\left\|T^{\prime}\right\|=\|T\|$.

We also use the following elementary result which holds for any $S$.

Proposition 2.2. Whenever $N \subset S$, there exists a simultaneous extension $F: C_{0}(N) \rightarrow C(S)$ with $\|F\|=1$. Moreover, if $S$ is locally compact, $F$ can be chosen with range in $C_{0}(S)$.

THEOREM $2.3[\mathrm{CH}]$. If $S$ is an $F$-space and $N \subset S$, there exists a simultaneous extension $E: C(N) \rightarrow C(S)$ with $\|E\|=1$. Moreover, if $S$ is locally compact then $E$ may be chosen with $E\left(C_{0}(N)\right) \subset C_{0}(S)$.

Proof. $N$ is normally embedded in $S$ by [GJ, 14N5]. Let $F$ be as in Proposition 2.2. Since $C_{0}(N)$ is separable, $F$ has an extension by Proposition 2.1 to $E: C(N) \rightarrow C(S)$ with $\|E\|=1$. Since $E \mid C_{0}(N)=F, E$ is a simultaneous extension by Corollary 1.3.

It is shown in [R] that, assuming [CH], $C(S)$ contains a subspace isometric to $(m)$ for every infinite $F$-space $S$. Since every infinite space $S$ contains a denumerable discrete subspace $[\mathbf{G J}$, p. 5], we note that this result also follows immediately from Theorem 2.3 .

For our last theorem we use the following, which is Theorem 1.3 of [B].

Proposition 2.4. If $N$ is closed in the locally compact space $S$ and if there exists a simultaneous extension $E: C(N) \rightarrow C(S)$ with $E\left(C_{0}(N)\right) \subset C_{0}(S)$, then $C_{0}(S)$ is not complemented in $C(S)$.

THEOREM $2.5[\mathrm{CH}]$. If $S$ is a locally compact F-space and $C_{0}(S)$ is complemented in $C(S)$ then $S$ is countably compact.

Proof. If not, $S$ contains a closed subspace $N$. The result follows by applying Theorem 2.3 and Proposition 2.4.

In [C, Theorem 2.7], Comfort shows that $S$ is countably compact whenever $S$ is a locally compact $F$-space and $\beta S-S$ is a retract of $\beta S$. The same conclusion also follows from [B, Theorem 2.2], whenever $S$ is a 
locally compact basically disconnected space and $C_{0}(S)$ is complemented in $C(S)$. Neither of these assume [CH]. Since every basically disconnected space is an $F$-space [GJ, 14N4] and $C_{0}(S)$ is complemented in $C(S)$ whenever $\beta S-S$ is a retract of $\beta S$, Theorem 2.5 , modulo [CH], improves both of these results.

\section{REFERENCES}

[B] H. Banilower, Projecting $C(S)$ onto $C_{0}(S)$, Proc. Amer. Math. Soc. 33 (1972), 349-354.

[C] W. W. Comfort, Retractions and other continuous maps from $\beta X$ onto $\beta X \backslash X$, Trans. Amer. Math. Soc. 114 (1965), 1-9. MR 32 \#3035.

[DS] N. Dunford and J. T. Schwartz, Linear operators. I: General theory, Pure and Appl. Math., vol. 7, Interscience, New York, 1958. MR 22 \#8302.

[G] D. B. Goodner, Subspaces of $C(S)$ isometric to $\mathrm{m}$, J. London Math. Soc. 3 (1971), 488-492.

[GJ] L. Gillman and M. Jerison, Rings of continuous functions, University Series in Higher Math., Van Nostrand, Princeton, N.J., 1960. MR 22 \#6994.

[H] W. Holstyński, Continuous mappings induced by isometries of spaces of continuous function, Studia Math. 26 (1966), 133-136. MR 33 \#1711.

[L] J. Lindenstrauss, On the extension of operators with range in a $C(K)$ space, Proc. Amer. Math. Soc. 15 (1964), 218-225. MR 29 \#5089.

[R] H. P. Rosenthal, On relatively disjoint families of measures, with some applications to Banach space theory, Studia Math. 37 (1970), 13-36. MR 42 \#5015.

Department of Mathematics, Bernard M. Baruch College, City University of New York, New York, New York 10010 